\title{
SUB-TENON'S VERSUS PERIBULBAR ANAESTHESIA FOR CATARACT SURGERY
}

\author{
M. C. BRIGGS ${ }^{1}$, S. A. BECK ${ }^{2}$ and L. ESAKOWITZ ${ }^{1}$ \\ Paisley
}

\begin{abstract}
SUMMARY
We present a comparison of one quadrant sub-Tenon's anaesthesia and peribulbar anaesthesia. Patient discomfort during injection of anaesthetic and during cataract surgery was assessed using a 10-point visual analogue scale ranging from no pain to the worst pain imaginable. Data are available for 74 patients undergoing cataract surgery under peribulbar anaesthesia and for 55 patients in whom sub-Tenon's anaesthesia was used. Pain scores for administration of anaesthetic were significantly lower (Kruskal-Wallis $H$-test, $p<0.01)$ for sub-Tenon's anaesthesia (mean 1.4) compared with the peribulbar technique (mean 2.4). However, a similar number of patients experienced pain of greater than 3 for the two techniques (10 $(18.5 \%)$ for sub-Tenon's and $14(18.9 \%)$ for peribulbar). Per-operative pain scores for sub-Tenon's anaesthesia (mean 0.5) were lower than those for peribulbar anaesthesia (mean 1.2) but not significantly so (Kruskal-Wallis $\boldsymbol{H}$-test, $\boldsymbol{p}=\mathbf{0 . 0 7 3}$ ). Significantly fewer patients, however, experienced pain of greater than 3 (Fisher exact test, $p<0.05$ ) in the sub-Tenon's group. In addition less anaesthetic solution and a shorter interval from administration to surgery was required in the subTenon's group. Sub-Tenon's anaesthesia appears to be a more effective method of anaesthesia than the peribulbar method.
\end{abstract}

A recent audit of cataract surgery in the UK indicated that almost $50 \%$ of such operations are performed under local anaesthesia. ${ }^{1}$ Several publications have recently investigated the merits of the various local anaesthetic techniques in use at present. These include retrobulbar and peribulbar, ${ }^{2}$ subconjunctival $^{3}$ and sub-Tenon's ${ }^{4}$ anaesthesia. As part of our continuing audit programme we performed an audit of the effectiveness of local anaesthesia for

From: ${ }^{1}$ Eye Department and ${ }^{2}$ Clinical Audit, Royal Alexandra Hospital, Paisley, UK.

Correspondence to: Mr Michael Briggs, St Paul's Eye Unit, Royal Liverpool University Hospital, Prescot Street, Liverpool L7 8XP, UK. cataract surgery in our department. The purpose of this audit was to assess the efficacy of local anaesthesia with regard to patient discomfort on administration of anaesthesia, during surgery and following surgery. No attempt was made to assess the efficacy of anaesthesia with regard to akinesia nor to assess the effect of local anaesthesia on amaurosis during surgery, post-operative recovery of vision, surgical outcome, or cosmetic effects such as subconjunctival haemorrhage and lid ecchymosis. This audit was performed in two cycles each lasting 1 month and conducted within an 18 month period. The period between the cycles coincided with a move among several of the staff in the department from using peribulbar anaesthesia to using the subTenon's method. As a consequence, in addition to gaining an indication of the efficacy of our local anaesthetic surgery we were able to make a direct comparison of peribulbar and sub-Tenon's anaesthesia in cataract surgery. The results of this comparison are presented.

\section{PATIENTS AND METHODS}

Two cycles of prospective audit were performed. For each cycle all patients having cataract surgery under local anaesthetic in the unit during a 4 week period were included in the audit. The first cycle was performed in October 1994 and the second in June 1995. No attempt was made to randomise patients. Surgeons chose the anaesthetic technique used. Between the first and second cycles several surgeons in the unit changed their preferred technique from a single infero-temporal peribulbar injection to the one-quadrant sub-Tenon's method. ${ }^{4}$ No additional oral or parenteral sedation or analgesia was used in the cases studied. Administration of local anaesthesia and cataract surgery was performed by all grades of staff: four consultants, one staff grade, one associate specialist and one registrar. In each cycle patient discomfort during administration of anaesthesia. during cataract surgery and post-operatively were

Eye (1997) 11, 639-643 Co 1997 Royal College of Ophthalmologists 


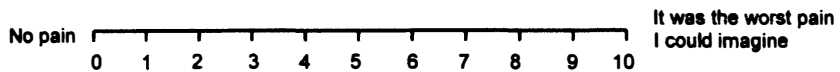

Fig. 1. Linear pain score chart.

assessed. In addition the method of administration of anaesthetic, the anaesthetic agents and volume used, surgeon administering the anaesthetic, the time between administration of anaesthetic and operation, the type of cataract surgery performed and administration of supplementary anaesthetic were all recorded.

To assess patient discomfort a $10 \mathrm{~cm}$ linear pain score chart was used (Fig. 1), on which patients were asked to indicate their discomfort on a scale from 0 (no pain) to 10 (worst pain imaginable). Patients were asked to score separately the discomfort of administration of anaesthetic, the discomfort of the operation itself and post-operative pain. The pain scores were completed by the patients under the supervision of a nurse on the ward on the day following surgery.

The sub-Tenon's method of administration of local anaesthesia used was as follows: The conjunctiva was anaesthetised with topical benoxinate $0.4 \%$. A small incision was made in the conjunctiva and Tenon's capsule 4-5 mm infero-nasal to the limbus with Wescott scissors. Moorfields' forceps were used to grip the conjunctiva and a blunt Southampton curved cannula passed into sub-Tenon's space. The cannula was passed posteriorly on sclera until its tip lay behind the equator where between 3 and $5 \mathrm{ml}$ was injected slowly. Gentle injection followed by advancement of the cannula in small steps was often necessary to hydrodissect Tenon's fascia from the sclera as the cannula was passed posteriorly.

\section{RESULTS}

Data are available on 76 patients for the first cycle and on 66 patients for the second. Administration of local anaesthesia was exclusively by peribulbar injection in cycle $1(n=76)$ and in 11 out of 66 cases $(17 \%)$ in cycle 2 . Administration was by subTenon's injection in 55 of 66 cases $(83 \%)$ in cycle 2. The results from cycle 1 are presented in comparison with the sub-Tenon's group in cycle 2 . The small number of patients in cycle 2 in whom peribulbar anaesthesia was used are not considered. For some of the analyses $n$ is less than either 76 or 55 because on some questionnaires these sections were not completed.

\section{Age and Sex}

Age of the patients ranged from 55 to 91 years in cycle 1 (mean 74.3 , median $75, n=75$ ) and from 56 to 88 years in the sub-Tenon's group (mean 74.3, median $74, n=55$ ). Female to male ratio was similar in the two groups, being $60 \%$ to $40 \%$ in cycle 1 and
$69 \%$ to $31 \%$ in the sub-Tenon's group. In all cases a combination of $2 \%$ lignocaine and marcaine (either $0.5 \%$ or $0.75 \%$ ) was used. Hyalase was used in all but 7 cases in cycle 1 and in all but 2 in the sub-Tenon's group.

\section{Volume of Anaesthetic}

In cycle 1 the range of volume of anaesthetic used (to the nearest milliltre) was 5-14 ml with a mean of 8.4 and median of $9(n=76)$. In the sub-Tenon's group the range was $2-5$ (mean 4.0 , median $4, n=55$ ). The volume of anaesthetic used was significantly lower for sub-Tenon's anaesthesia compared with the cases in cycle 1 in which peribulbar anaesthesia was used (Kruskal-Wallis $H$-test, $p<0.0001$ ).

\section{Staff Administering Anaesthetic}

Anaesthetic was administered by a consultant ophthalmologist in $56 \%$ of cases in cycle 1 and in $20 \%$ of cases in cycle 2 .

\section{Interval from Administration of Anaesthetic to Surgery}

In cycle 1 the interval between administration of local anaesthetic and surgery ranged from 6 to 15 minutes with a mean of 10.2 minutes and median of $10(n=73)$. The equivalent times for the sub-Tenon's patients in cycle 2 were a range of 4 to 8 , a mean of 5.0 and a median of $5(n=54)$. The time interval from administration of anaesthetic to surgery for subTenon's anaesthesia was significantly shorter than that in cycle 1 (Kruskal-Wallis $H$-test, $p<0.0001$ ).

\section{Supplementary Anaesthetic}

Five patients received supplementary anaesthetic in cycle 1 , as did 1 patient in cycle 2 . This was not of statistical significance (Fisher exact test, $p=0.20$ ).

\section{Pain on Administration of Anaesthetic}

Pain scores for administration of anaesthetic were as follows. In cycle 1 the range was $0-10$, the mean 2.4 and the median $2(n=74)$. For the sub-Tenon's method in cycle 2 the range was $0-9$, the mean 1.4 and the median $0 \quad(n=54)$. There was thus a significant difference in the pain scores recorded when the sub-Tenon's group is compared with the patients in cycle 1 who all had peribulbar injections (Kruskal-Wallis $H$-test, $p<0.01$ ). These pain scores for the peribulbar group in cycle 1 and for the subTenon's group in cycle 2 are illustrated graphically in Fig. 2. Fourteen patients $(18.9 \%, n=74)$ in cycle 1 and $10(18.5 \%, n=54)$ of those in the sub-Tenon's group in cycle 2 experienced pain which they scored at greater than 3 . 


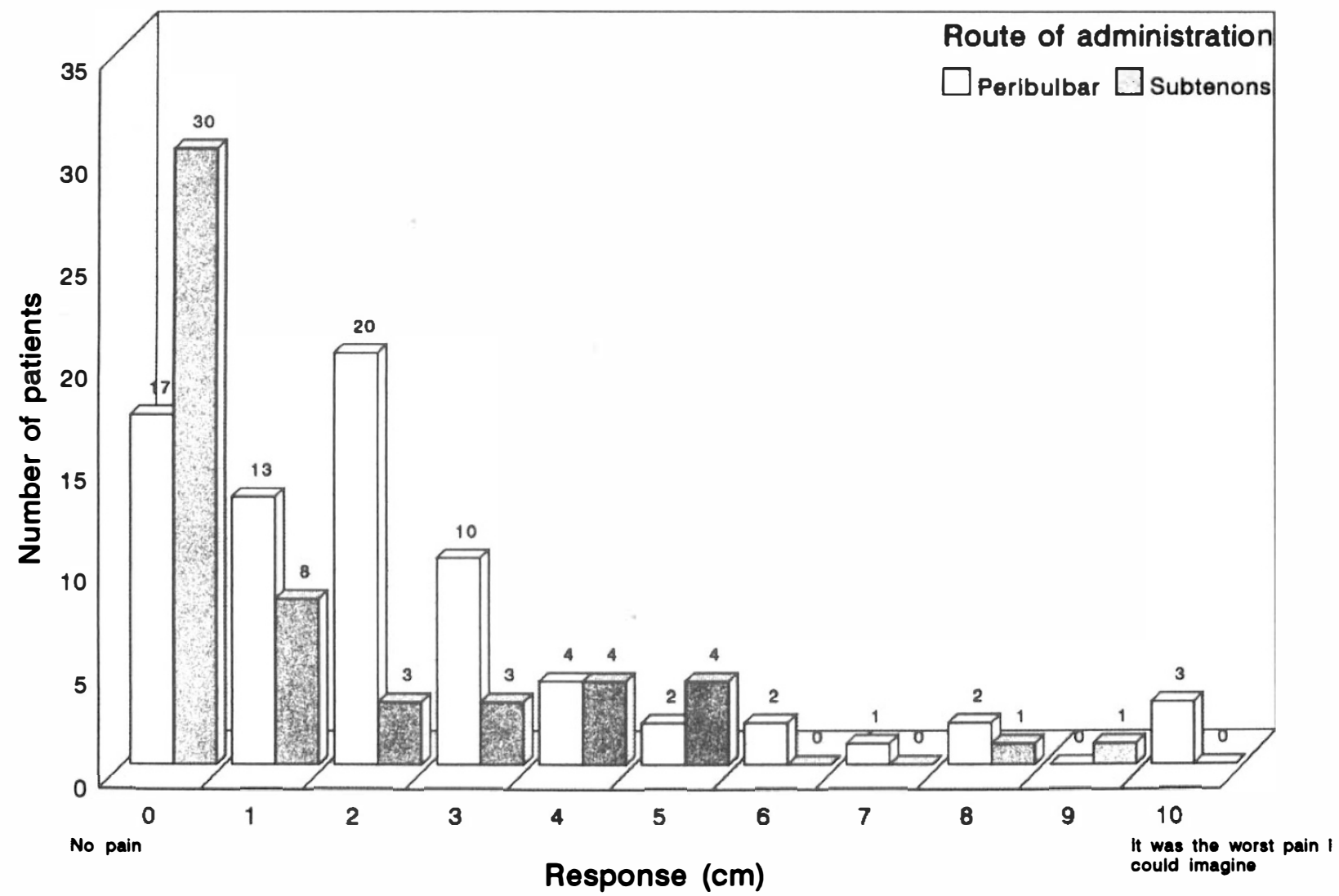

Fig. 2. Pain of administration of local anaesthetic. Patients were asked: 'How much did this injection hurt?'

\section{Pain of Operation}

For per-operative pain the range of scores for cycle 1 was 0 to 10 with a mean of 1.2 and a median of 0 $(n=74)$. For those patients given sub-Tenon's anaesthesia the scores ranged from 0 to 4 with a mean of 0.5 and a median of $0(n=55)$. Pain levels are higher but the difference is not statistically significant when those patients undergoing cataract surgery with peribulbar anaesthesia in cycle 1 are compared with those undergoing cataract surgery with sub-Tenon's anaesthesia (Kruskal-Wallis $H$-test, $p=0.073)$. These pain scores for cycle 1 and for the peribulbar group and for the sub-Tenon's group in cycle 2 are illustrated graphically in Fig. 3. The number of patients grading operative pain at greater than 3 is $8(10.8 \%, n=74)$ in cycle 1 and 1 $(1.8 \%, n=55)$ in those given sub-Tenon's anaesthesia in cycle 2 ; this difference is statistically significant (Fisher exact test, $p<0.05$ ).

\section{Post-operative Pain}

Post-operative pain ranged from 0 to 10 in cycle 1 with a mean of 1.7 and median of $1(n=74)$. For the sub-Tenon's patients in cycle 2 the range was 0 to 5 , the mean 1.2 and the median $1(n=55)$. There was no statistically significant difference in the postoperative pain scores for the two methods of anaesthesia (Kruskal-Wallis $H$-test, $p=0.41$ ).

\section{Complications}

No significant complications such as globe perforation, optic nerve injury or systemic side-effects occurred in either the peribulbar group or the subTenon's group.

\section{DISCUSSION}

The technique of peribulbar anaesthesia has recently been preferred to retrobulbar anaesthesia as it is associated with a smaller risk of globe perforation, retrobulbar haemorrhage, optic nerve damage and injection of the anaesthetic solution into the subarachnoid space. ${ }^{5}$ However, the peribulbar method itself is not absolutely safe, as has been reported frequently. ${ }^{6,7}$ Subconjunctival anaesthesia ${ }^{3}$ is a further effective and safer alternative; however, this technique provides no akinesia. Sub-Tenon's anaesthesia as described by Hansen et al. ${ }^{8}$ and by Stevens ${ }^{4}$ has recently become popular as it provides effective anaesthesia and akinesia without the risks associated with retrobulbar and to a lesser extent peribulbar anaesthesia.

The purpose of audit is to assess the efficacy of current clinical practice and to determine how that practice can be improved. In this case the aim was to determine the efficacy of our provision of local anaesthesia for cataract surgery in terms of patient discomfort. The aim of local anaesthesia must be to 


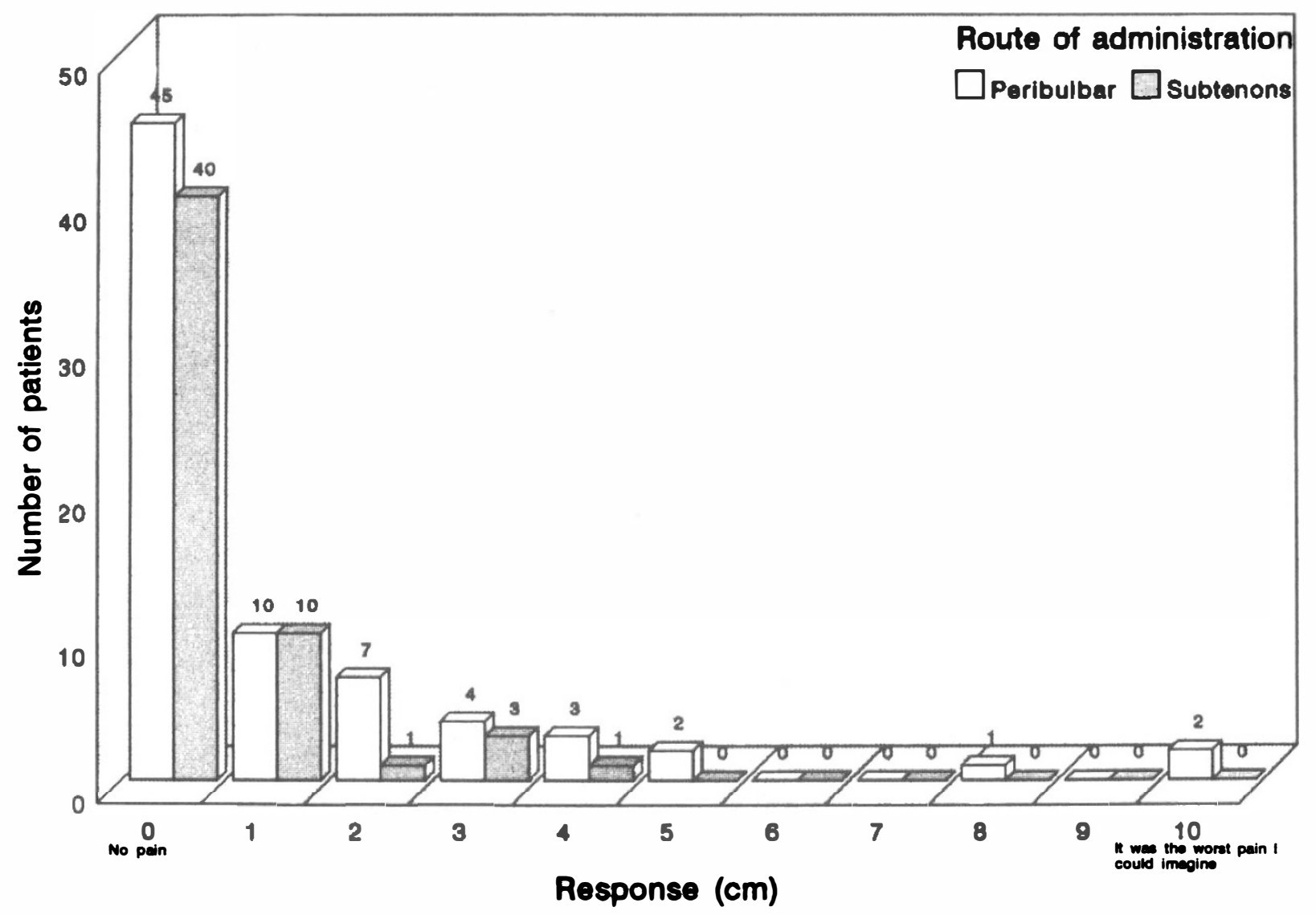

Fig. 3. Pain experienced during surgery. Patients were asked: 'How much pain did you feel during your operation?'

achieve as low a level of discomfort during surgery as possible. Administration of the anaesthetic will inevitably be associated with some discomfort but an ideal technique should minimise this. Use of the sub-Tenon's method appears on the basis of these results to significantly reduce the overall level of discomfort associated with administration of local anaesthesia when compared with the peribulbar method. Setting a target pain score of 3 or less, however, our results demonstrate that there is a large minority of patients who experience significant discomfort during administration of both types of local anaesthesia. We might consider that the failure rate in terms of achieving minimum discomfort for injection of the anaesthetic is $18.9 \%$ in the case of peribulbar anaesthesia and $18.5 \%$ in the case of subTenon's anaesthesia. Our figures for the sub-Tenon's patients compare with those in a study by Stevens ${ }^{4}$ who reported a median score of 1 and a mean of 1.0 for administration of sub-Tenon's anaesthesia (a 10-point scale was used with ' 10 ' described as 'unbearable pain'). However, in that study no patient reported a score of greater than 5 for administration of anaesthetic. In his comparison of peribulbar and retrobulbar techniques. Murdoch ${ }^{2}$ reported a mean pain score of 4.8 for administration of peribulbar anaesthesia ('10' described as 'severe pain').
Although there is trend for a lower range of pain scores for per-operative discomfort for the group of patients given sub-Tenon's anaesthesia when compared with those in cycle 1 , the difference is not statistically significant. There were, however, significantly fewer patients experiencing pain graded at greater than 3 . The failure rate, defined as a peroperative pain score of greater than 3 , is $10.8 \%$ for the peribulbar group and $1.8 \%$ for the sub-Tenon's group. In this study, therefore, the failure rate for prevention of per-operative discomfort is significantly lower for the sub-Tenon's method than for the peribulbar method.

Our study suggests that post-operative pain levels are not influenced by the method of anaesthesia. In addition to the findings with regard to the level of discomfort experienced by the patients we found that significantly less anaesthetic solution was required to provide anaesthesia and akinesia to the satisfaction of the surgeon when the sub-Tenon's method was used in comparison with the peribulbar technique. The interval between administration of the anaesthetic and the time at which the surgeon was confident that anaesthesia was adequate to commence the operation was also significantly shorter when the sub-Tenon's technique was used. 
In addition to the findings of this study it is likely that sub-Tenon's anaesthesia offers a significantly reduced risk of complications such as scleral perforation, retrobulbar haemorrhage, optic nerve injury and injection of anaesthetic solution into the subarachnoid space, as no sharp instrument is passed into the orbit. The number of patients involved in this study is too small to demonstrate any difference in the complication rate between the two techniques and it is likely that only widespread and prolonged use of this relatively new method of local anaesthesia will show conclusively that there is a lower risk of these complications.

In conclusion, the results of this audit demonstrate that the sub-Tenon's method of administration of local anaesthesia for cataract surgery is more effective than the peribulbar technique, with significantly fewer patients experiencing unacceptable levels of pain. It is significantly less uncomfortable on administration than the peribulbar method and reduces the interval between administration of anaesthesia and surgery.
Key words: Sub-Tenon's, Peribulbar, Cataract surgery, Local anaesthesia, Pain scores.

\section{REFERENCES}

1. Courtney P. National cataract surgery survey. Eye 1992;6:487-92.

2. Murdoch IE. Peribulbar versus retrobulbar anaesthesia. Eye 1990;4:445-9.

3. Redmond RM, Dallas NL. Extracapsular cataract extraction under local anaesthesia without retrobulbar anaesthesia. Br J Ophthalmol 1990;74:203-4.

4. Stevens JD. A new local anaesthetic technique for cataract extraction by one quadrant sub-Tenon's infiltration. Br J Ophthalmol 1992;76:670-4.

5. Davis DB, Mandel MR. Posterior peribulbar anaesthesia: alternative to retrobulbar anaesthesia. J Cataract Refract Surg 1986;12:182-4.

6. Mount AM, Seward HC. Scleral perforations during peribulbar anaesthesia. Eye 1993;7:766-7.

7. Hawksworth NR. Peribulbar anaesthesia [letter]. Br J Ophthalmol 1992;76:254.

8. Hansen EA, Mein CE, Mazzoli R. Ocular anaesthesia for cataract surgery: a direct sub-Tenon's approach. Ophthalmic Surg 1990;21:696-9. 